\title{
SOBRE LA ESTRUCTURA DEL ELECTRÓN: UNA REFLEXIÓN EN TORNO A LOS MODELOS DE LORENTZ Y POINCARÉ.
}

\section{ON THE STRUCTURE OF THE ELECTRON: A REFLEXIÓN AROUND THE MODELS OF LORENTZ AND POINCARÉ.}

\author{
Mauricio Rozo Clavijo ${ }^{1}$ \\ Wilmer Ferney Melo Bernal ${ }^{2}$
}

\begin{abstract}
Resumen
En este trabajo se realiza una exploración sobre los textos originales de Lorentz y Poincaré alrededor de la estructura del electrón, con el objetivo de estudiar su estructura y analizar la posibilidad de explicar la naturaleza de su masa como un efecto electrodinámico. Bajo este contexto, se considera al electrón no como una carga puntual, sino como una estructura de simetría esférica de radio diferente de cero. En este sentido, surge un modelo sobre el electrón que está en correspondencia con los datos empíricos observados. Por otro lado, en la enseñanza de la física se reconoce abiertamente que se necesita el apoyo de información visual dirigida a los estudiantes, buscando favorecer su comprensión en los fenómenos abordados. Frente a esto es necesario hacer evidente que toda representación es una manera de interpretar y significar los fenómenos del mundo físico.
\end{abstract}

Palabras clave: Electrón, Estructura, masa electromagnética, representación.

\section{Abstract}

This paper is based on the exploration of original projects undertaken by Lorentz and Poincaré on the structure of the electron. It is also aimed at analyzing the possibility of explaining the nature of its mass considering it as an electromagnetic effect. Through this paper, we examine the electron as a spherical structure with different of zero radiuses. Thus, the authors of this paper searched for a specific model of the electron which eventually may match to the empirical data observed giving account for the comprehension of this particular phenomena scopes.

Keywords:Electron Structure, electromagnetic mass, representation.

\section{Introducción.}

En el contexto de la enseñanza de la física los docentes son consientes de los problemas educativos que surgen en las aulas frente a la complejidad y diversidad de tópicos abordados. En este sentido cabe reflexionar sobre las temáticas desarrolladas en las aulas de clase, en torno a cómo aprenden los estudiantes y cuales modelos

\footnotetext{
${ }^{1}$ Universidad Pedagógica Nacional, mclavijo@pedagogica.edu.co

${ }^{2}$ Universidad Pedagógica Nacional, wmelo91@gmail.com
} 
explicativos y representaciones se utilizan para el proceso de enseñanza aprendizaje de la física en la escuela.

Reconocerlo hace necesarioque se genere un dialogo permanente y en constate evaluación entre lo que se enseña y las prácticas de enseñanza [García, A. 2009].En este sentido, se pretende que los estudiantes le tomen sentido y significado a las magnitudes o tópicos abordados para su enseñanza.

Aceptando que en la enseñanza de la física se necesita el apoyo de información visual dirigida a los estudiantes, es necesario reflexionar entorno a la pertinencia de la informaciónque se presenta,buscando favorecer con dicha información la comprensión de los fenómenos en estudio [Álvarez, T. 2010].

La información que se presenta a través de esquemas, gráficos, dibujos, etc., complementan la exposición del docente y facilitan representaciones de los conceptos presentados. Estos recursos juegan un papel importante en el proceso de aprendizaje de los estudiantes aunque en algunas ocasiones no se explicite que son solo modelos, razón por la cual los estudiantes tienden a atribuir esas estructuras y representaciones a las realidades del mundo físico.

La enseñanza de la masa del electrón y su estructura, tradicionalmente no son temáticas analizadas en los cursos de electromagnetismo a nivel universitario.[Gramajo, 1993] para poder desarrollar dichos tópicos, se hace necesario el uso de representaciones que conducen a formular un modelo explicativo sobre el fenómeno físico abordado. Aunque se presentan algunos inconvenientes de significación de las representaciones, estas facilitan el aprendizaje de conceptos que requieren de un alto nivel de abstracción.

Para hacer inteligibles las reflexiones en torno a la estructura del electrón, a continuación se plantean los problemas inmersos en la electrodinámica clásica que tradicionalmente no son abordados en su enseñanza.

\section{Energíay Momentum de una carga puntual.}

Al tomar conceptos propios de la mecánica y adoptar también algunas de sus representaciones, la teoría electromagnética presenta problemas como son, los resultados que se obtienen al calcular la energía y la intensidad de campo eléctrico de una partícula cargada. Tomando un modelo mecanicista, un electrón puede ser considerado como una distribución superficial esférica de carga de radio $\boldsymbol{a}$;modelo que presenta problemas cuando se desea calcular la intensidad de campo eléctrico y su energía cuando $\boldsymbol{a}=\mathbf{0}$.En este caso la energía del campo eléctrico está dada por:

$$
U=\int_{r=0}^{\infty} \frac{q^{2}}{32 \pi^{2} \epsilon_{0} r^{4}} 4 \pi r^{2} d r=\int_{r=0}^{\infty} \frac{q^{2}}{8 \pi \epsilon_{0} r^{2}} d r=-\left.\frac{q^{2}}{8 \pi \epsilon_{0}} \frac{1}{r}\right|_{r=a} ^{r=\infty},
$$

Presentando una energía infinita. Este resultado es incompatible con las evidencias experimentales, ya que estos efectos no son observados.

Por otra parte al considerar al electrón en movimiento con una velocidad constante $v \ll c$ su momentum, el momentum del campo electromagnético, está dado por [Feynman, 1963]: 
5o CONGRESO NACIONAL DE ENSEÑANZA DE LA FÍSICA.

Universidad Pedagógica Nacional (U. P. N)

Universidad Distrital Francisco José de Caldas (U. D. F. J. C)

Bogotá, Colombia. 16 al 20 de mayo 2011

$p=\frac{2}{3} \frac{q^{2}}{4 \pi \epsilon_{0}} \frac{v}{a c^{2}}=\frac{2}{3} \frac{e^{2}}{a c^{2}} v$,

siendo proporcional a la velocidad $v$. Comparando la ecuación (2) con la definición de momentum clásico $\boldsymbol{p}=\boldsymbol{m} \boldsymbol{v}$, la masa de la partícula, queda dada por:

$$
m_{\text {elec }}=\frac{2}{3} \frac{e^{2}}{a c^{2}},
$$

denominada masa electromagnética [Langevin P].

En este orden de ideas, surge la motivación de plantear la pregunta, de si la masa de un cuerpo cargado es un efecto puramente electromagnético. Es importante resaltar que la masa electromagnética definida por la ecuación (3) se hace infinita considerando la carga puntual, $\boldsymbol{a}=\mathbf{0}$.

Los resultados anteriores tienentanto base experimental como teórica, ya que los experimentos de Kaufmann sobre la desviación eléctrica y magnética de los rayos beta, conducen a considerar que la masa de un electrón depende únicamente de su velocidad y tiene un origen completamente electromagnético. [Ritz 1980 ].

En este sentido, se ve la necesidad de atribuir una estructura al electrón; Pues como lo presenta Maxwell partiendo del análisis de la fuerza eléctrica que ejercerían los elementos de carga dela superficie de un punto cargado, "un punto cargado con una cantidad finita de electricidad no puede existir en la naturaleza" [Maxwell,1873] aunque reconoce que es conveniente hablar de puntos electrificados, los cuales deben ser pensados como pequeños cuerpos cuyas dimensiones sean insignificantes en comparación con las distancias en las que se están analizando las interacciones.

\section{La inercia electromagnética y la estructura del electrón:modelo de Languevin.}

Para Languevin la inercia no es una propiedad fundamental de la materia, ya queen sus diversas definiciones, la masa como coeficiente de inercia, la masa como capacidad de impulso y la masa como capacidad de energía cinética, dejan de coincidir cuando la velocidad del cuerpo se aproxima a la velocidad de la luz $\boldsymbol{v} \approx \boldsymbol{c}$. En este sentido, para un mismo cuerpo se tiene diferentes valores de su masa en función de la velocidad.

Reconociendo las limitaciones inmersas en mecánica clásica (acción a distancia, e interacción instantánea), Languevin supone que la teoría electromagnética es más viable para la explicación de los fenómenos.En este sentido,buscaunainterpretación electromagnética de la inercia de las partículas cargadas, aunque reconoce que tal síntesis electromagnética puede ser imposible.

Basado en los resultados experimentales de Rowland.[Languevin P.], le asocia una estructura al electrón, considerándolo como una esfera de radioa y de carga emoviéndose uniformemente.Adoptando la hipótesis de Abraham.[Languevin P.], de indeformabilidad para la partícula esférica, esta conserva su forma para cualquier velocidad.Además, considera que la esfera solo está cargada en su superficie y que no produce campo magnético ni eléctrico en su interior. En el caso de $\boldsymbol{v} \ll \boldsymbol{c}$ el campo 
5o CONGRESO NACIONAL DE ENSEÑANZA DE LA FÍSICA.

Universidad Pedagógica Nacional (U. P. N)

Universidad Distrital Francisco José de Caldas (U. D. F. J. C)

Bogotá, Colombia. 16 al 20 de mayo 2011

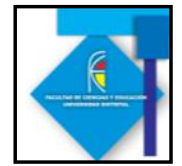

eléctrico mantiene su distribución entorno a la esfera con líneas de fuerza radiales simétricamente dispuestas a su alrededor.

Realizando un análisis de la energía del campo electromagnético, Langevin obtiene como resultado una inercia de origen electromagnético, la cual es función de la magnitud de la carga y del radio de ésta. Razón por la cual expone que "toda variación de la carga o del radio y, por ende, de la energía almacenada alrededor de la esfera, implica una variación proporcional de su inercia" [Langevin].Interpretándose este resultado como una inercia adicional a la inercia mecánica.

Aunque los trabajos de Langevin contribuyeron a dar respuesta sobre la estructura del electrón, siguen presentándose inconsistencias con los datos experimentales y con las teorías sobre el movimiento de los cuerpos. En este sentido, podemos citar a Lorentz: "se puede formular cualquier modelo de las propiedades y formas de los electrones, enfocados en buscar el que mejor se adecue a estos, el que mejor reproduzca las propiedades observadas de los electrones".[Lorentz,1927].

\section{El modelo de Lorentz.}

En contraste con las ideas de Langevin, Lorentz propone que los cuerpos cambian su forma en ladirección de su movimiento. Por lo cual, el electrón en movimiento adoptara una forma elipsoidal dada por la siguiente ecuación,

$$
\frac{x^{\prime 2}}{\gamma^{2}}+y^{\prime 2}+z^{\prime 2}=l^{2} r^{2}
$$

con la idea de quela carga sea un invariantedurante el movimiento y siendo $r$ el radio del electrón. Para Lorentz, el electrón en reposotiene simetría esférica, sin embargo cuando se encuentra en movimiento adquiere una simetría elipsoidal cuyos semiejes son $r / l \gamma, r / l, r / l$.

\section{Modelo de Poincaré.}

Poincaré admite y postula la localización de la energía en los campos eléctricos y magnéticos fuera de la materia, con el fin de mantener la validez del principio de conservación de la energía[Poincaré, 1906]. "La energía se localiza principalmente en las partes del éter más cercanas al electrón". A puntos distantes se puede considerar al electrón como una estructura puntual.

Partiendo de la existencia de corpúsculos con carga negativa, Poincaré reconoce la dificultad sobre la estructura del electrón. Sin embargo, argumenta que existen fuerzas de naturaleza diferente a las fuerzas electromagnéticas presentes en el electrónpara darle estabilidad.Dichas fuerzas se establecen conjuntamente en el campo eléctrico circundante y deben contrarrestar la repulsión electrostática, impidiendo la dispersión de la carga. Introduciendo una magnitud denominada, presión de Poincaré, él muestra como estas fuerzas actúan conjuntamente.

Considerando el electrón en reposo y su carga distribuida sobre la superficie de una esfera de radio $\boldsymbol{r}=\boldsymbol{a}$,las acciones de repulsión electromagnéticas en pares entre los diversos elementos de carga, se expresan como repulsión electrostática, orientada hacia el exterior de la capa de densidad superficial de carga. Repulsión que es equilibrada si el electrón está sometido a una presión que provenga, en palabras 
textuales "del éter exterior". Logrando estabilidad en dicho equilibrio, como si cada electrón fuera hueco y sujeto a una presión inercial constante [Langevin].

Por otro lado, si el electrón se encuentra en movimiento el efecto de la presión exterior y de la fuerza electromagnética, hace que la forma de equilibrio cambie y se convierta en un elipsoide achatado (modelo de Lorentz). "el electrón se achata en la dirección de su movimiento hasta encontrar equilibrio".

La presión exterior que brinda estabilidad al electrón, para el caso del reposo, está dada por la ecuación,

$$
\varsigma=\frac{e^{2}}{8 \pi K_{o} a^{4}}
$$

siendo $K_{0}$ el poder inductor del vacío [Langevin].

Considerando el modelo general, queincluya los de Abraham, Langeviny Lorentz como casos especiales: los semiejes del electrón en reposo son:

$$
r, \theta r, \theta r
$$

y los del electrón en movimiento son, [Schwartz, 1971]

$$
\gamma l r, l \theta r, l \theta r
$$

Los tres modelos especiales bajo consideración son entonces dados por las siguientes determinaciones:

$$
\begin{array}{ccc}
r=\text { const }, \quad \theta=1 & (\text { Abraham }) \\
l=\gamma^{-\frac{1}{3}}, \quad \gamma l r=\text { const }, \quad \theta=\gamma & (\text { Langevin }) \\
l=1, \quad \gamma r=\text { const }, \quad \theta=\pi \gamma & (\text { Lorentz }) .
\end{array}
$$

Hasta este punto se presentaron algunas reflexiones alrededor de los trabajos de Langevin, Lorentz y Poicarésobre la estructura del electrón. Cabe preguntarse sobre cuáles representaciones se podrían abordar para la enseñanza en la escuela y cuáles son los modelos mentales que estas representaciones propician para la interpretación y asimilación de dichos tópicos. Reflexión que queda abierta y que puede ser alimentada por las experiencias de aula al introducir los diferentes modelos sobre la estructura del electrón en las clases de ciencias.

\section{Bibliografía.}

Alvarez, T. (2010). La visualización de conceptosmatemáticos y el aprendizaje del electromagnetismo. Latín American Journal of Physics Education. Vol 4, No. 1.

Colombia, Universidad Pedagógica Nacional.(199). El concepto de carga eléctrica en una concepción de física clásica de campos. Las propuestas de Faraday, Maxwell y Hertz. Bogota: M. Gramajo.

Feynman. R. (1964) 'The Feynman lectures on Physics, Mainly Electromagnetism and Matter, Volume II. Masachusetts, E.U. Addison- Wesley Publishing Company. 
García, A. (2009). Investigación en didáctica de la física: tendencias actuales e incidencia en la formación del profesorado. Latín American Journal of Physics Education. Vol 3, No. 2.

Langevin P. (). La inercia de la energía y sus consecuencias. Introducción a la Relatividad, Cap 3.

Lorentz. H, (1927) Problems of modern physics. California: Ginn and Company.

Maxwell J. C (1873). A treatise on electricity and magnetism.Oxford: Claredom Press.

Poincaré. (1906) Sur le dynamique de l'électron. Rediconti del circolo matematico di Palermo.

Ritz. W (1980). Electrodynamics mass.Recherches critiques sur l'Electrodynamique Générale. Annales de Chimie et de Physique, Vol. 13.p,145.

Schwartz. H. (1971) Poincare's rediconti paper on relativity. En J. Hsu.(Ed) Lorentz and Poincaré invariance: 100 years on relativity. (pp.76-115) Singapore: World Scientific Publishing. 